\title{
Genetic Evidence Disapproves Coevolution between O.sinensis and Host Insects from Qing-Tibetan Plateau
}

\section{Zhifan Guo}

Yunnan University https://orcid.org/0000-0002-7979-1364

\section{Xiang Li}

Yunnan University

\section{Tao Sha}

Yunnan University

Yan Li (D liyan0910@ynu.edu.cn )

Yunnan University https://orcid.org/0000-0001-8643-4931

\section{Original article}

Keywords: co-evolution, Ophiocordyceps sinensis, ITS, COI

Posted Date: June 23rd, 2021

DOI: https://doi.org/10.21203/rs.3.rs-593174/v1

License: (9) This work is licensed under a Creative Commons Attribution 4.0 International License. Read Full License 


\section{Abstract}

Host-parasite coevolution is driven by the selective pressures each partner conferring on the other to merely maintain parity with other evolving species. Ophiocordyceps sinensis represents one of excellent model host-parasite complex, allowing us to explore evolutionary relationship between host and parasite. Although previous studies have demonstrated coevolution between 0 .sinensis and host insects, the incongruence in phylogenetic relationship of both is incompatible. In this study, we used ITS gene of O.sinensis and $\mathrm{COI}$ gene of host insects to evaluate the phylogenetic relationship, estimate the divergence time, infer demographic history, analyze identity by state distance matrix, and simultaneously explore evolutionary relationship or address the evolutionary pattern. We found distinct phylogenetic topology between 0 .sinensis and host insects from the main distribution of China. Additionally, the branch length ratio, demographic inference, and IBS analysis consistently suggested independent evolution between 0. sinensis and host insects rather than arms race coevolution. However, populations of both 0 . sinensis and host insects from Qinghai lake showed similar evolution rate and phylogenetic topology, which might be associated with the formation of Qinghai Lake. Together, the data presented here provided new insights into host-parasite evolutionary relationship.

\section{Key Points}

O.sinensis and host insects evolve across the main land of Tibetan Plateau with only exception found in Qinghai Lake.

The differentiation of 0 .sinensis is at least partially associated with uplift of Tibetan Plateau.

\section{Introduction}

Host-parasite coevolution is a special form of interactions including reciprocal adaptive genetic changes in the particular host and parasite species. It is driven by the selective pressures each partner conferring on the other, in the form of evolutionary arms race (Milutinovic et al. 2016; Neiman and Fields 2016).

Parasitic fungi and their particular host insects are the most pervasive coevolution pattern detected in natural world (Joop and Vilcinskas 2016). For example, Tribolium castaneum is resistant to Beauveria bassiana infection by secreting microbicidals (Pedrini et al. 2015); Drosophila melanogaster exits substantial genetic variation of in resistance to infection of Entomophthora muscae (Wang et al. 2020).

One of the most prominent parasitic fungi is Ophiocordyceps sinensis (syn. Cordyceps sinensis), a genus of ascomycete (Sung et al. 2007). It parasitizes mainly on the larvae of Thitarodes and converts it into sclerotium, from which fungus fruiting body grows (Wang and Yao 2011; Zhang et al. 2012). Arms race is believed to occur between 0 .sinensis and its host, due to the comparable correlations between genetic distance and latitude-dependent distribution of both fungi and host, using ITS sequences of O. sinensis and $\mathrm{CO}$ / sequences of the host insects from the same complex of host cadaver and stroma (Quan et al. 2014b; Zhang et al. 2015). Alternatively, neutral evolution could drive similar 
correlation between genetic distance and geographic distance in both host-parasite species. Moreover, this fungal invasion occurs rarely in nature, which succeeds only moderately in experimental conditions via multiple assisted methods (Li et al. 2019; Peng et al. 2013). Reciprocal selective forces should hardly influence the whole populations, let alone drive arms race. Therefore, it was reasonable to interrogate the driving force of interactions during the evolution of 0 .sinensis and Thitarodes each.

\section{Materials And Methods}

\section{Samples}

Here, we collected 29 Ophiocordyceps sinensis samples from Qinghai, and Yunnan. A 504 bp length polymerase chain reaction product within ITS region was amplified for 0 .sinensis using primers designed in previous research (White et al. 1990) (5'-F: TCCTCCGCTTATTGATATGC; 3'-R: GGAACAATGCTGAAAATGAAGG). We also obtained 617 bp length COl sequences from corresponding host insects with our designed primers (5'-F: AATTGGTTAAATTCTACTAATG; 3'-R: CAATAGGAGGAAATCGACCTT). For specified topology and wider coverage in geographic analysis, we combined an additional 33 ITS and $33 \mathrm{CO} /$ sequences downloaded from NCBI website, with accession Nos. listed Table S1. The integrated samples covered the main habitats in China (Fig. 1a).

\section{Phylogenetic analysis}

These sequences were assembled and checked with SeqMan7.1 (Burland 2000). All of them were analyzed in MEGAX including alignment, computing pairwise distances and constructing the phylogenetic tree using maximum-likelihood method based on Kimura 2-parameter model (bootstrap 1000) (Edgar 2004; Kimura 1980; Kumar et al. 2018).

\section{IBS analysis between fungal and host insects}

We used the pheatmap package in R to draw the heatmaps of ITS and COI genes based on the IBS distance matrix to show the IBS correlation within each fungal sample and corresponding infected insect and observe the differentiation within and among different groups of 0 .sinensis and host insects (Tibetan Plateau, Qinghai Lake, Nyingchi, Yunnan). Then we conducted correlation analysis using Spearman method in $R$ to observe the correlation of IBS distance between $O$.sinensis and corresponding host insect.

\section{Analysis of the genetic structure}

After alignment, we used the software DNAsp v6 to calculate the DNA polymorphism and visualize the single nucleotide polymorphism (SNP) sites (Rozas et al. 2017). Principle component analyses were performed using GenAIEx v6.502 through the SNP of ITS and COI sequences (Peakall and Smouse 2012).

\section{Divergence time estimates}


To estimate the divergence time of either $O$. sinensis and host insects, we used the Bayesian method implemented in the program BEAST2 v2.6.2 (Barido-Sottani et al. 2017; Bouckaert et al. 2019).

For analysis of 0 .sinensis, the fossil evidence of Paleoophiocordyceps coccophagus (99-105million years ago, Mya) was used as the crown calibration point (Kumar et al. 2017; Sung et al. 2008; Zhang et al. 2015). For host insects, we used a standard insect mtDNA clock of $2.3 \%$ pairwise sequence divergence per million years because there are no fossil records or unambiguous biogeographic events that could be used to calibrate the host insect tree (Brower 1994; Zhang et al. 2015).

The relaxed uncorrelated lognormal clock model and the strict clock model was used for the fungus data set and the host insect data set, respectively (Zhang et al. 2015). We set the Calibrated Yule model for fungus data set (Heled and Drummond 2012) and Coalescent Constant Population for the host insect data set as the tree priors (Heled 2012). Markov Chain Monte Carlo (MCMC) analyses were run for 10 million generations with parameters sampled every 2000 generations. Convergence of the runs was assessed using Tracer v1.7.1 to analyze the output of BEAST, which indicated most parameter valued had effective sample size well above 200 (Rambaut et al. 2018). Then TreeAnnotator v2.6.2 was used to calculate the mean ages of all the nodes, the corresponding $95 \%$ highest posterior density interval (HPD) ranges and the posterior clade probability for each node with the target tree type of Maximum clade credibility tree (Bouckaert et al. 2014). Finally, the chronogram was visualized using FigTree v1.4.4 (http://tree.bio.ed.ac.uk/software/figtree/).

\section{Demographic inference}

The historical population dynamics of 0 .sinensis and host insects were inferred by coalescent-based Bayesian skyline plots (BSP) implemented in BEAST2 v2.6.2 (Bouckaert et al. 2019; Drummond et al. 2005). To plot the population history, load the log file in Tracer v1.7 (Rambaut et al. 2018) and select the menu Analysis/Bayesian Skyline Reconstruction.

\section{Results}

\section{Genetic structure and phylogenetic relationship}

Non-parameter principal component analysis (PCA) manifested four genetic clusters for stroma samples (O.sinensis) corresponding to their geographic distribution: Tibetan Plateau group except Nyingchi (TPo), Qinghai Lake group (QLo), Yunnan group (YNo), and Nyingchi group (NCo) (Fig. 1b). This geographicdependent separation remained partially in host samples (Thitarodes), such as Qinghai Lake group (QLt) and Nyingchi group (NCt). Exceptions were Yunnan group (YNt) and Tibetan Plateau group expect Nyingchi (TPt), which clustered with each other thoroughly (Fig. 1c). A parallel affinities were recovered in phylogenetic trees constructed via maximum-likelihood (ML) methods, using Ophiocordyceps irangiensis and Ogygioses as outgroup for stroma and host, respectively (Fig. 1d-e). 
Coevolution between parasite and host is defined as the extent to which their phylogenetic trees are congruent (Legendre et al. 2002). In spite of several researches that demonstrated coevolution between $O$. sinensis and host insects (Zhang et al. 2015), we observed incongruous phylogenetic relationship within the complex of host cadaver and stroma. Specifically, stroma phylogeny inferred a close relationship between YNo and NCo, which formed a sister branch with TPo and QLo (Fig. 1d). Meanwhile, YNt clustered with TPt, which formed a sister branch with QLt. NYt was basal to all of them (Fig. 1e). Considering the geographic distance and the mobility of insects, this incongruence may infer a mixture event between YNt and TPt. Parasite and corresponding host driven by arms race should experience similar divergence time. However, the branch length ratio of TPo/NCo (1.04) was nearly 2 times to that of TPt/NCt (0.53), indicating different evolutionary trajectories with different time-scale in each O.sinensis and Thitarodes.

\section{Divergence time}

To infer the causative factors that promoted divergence in 0 .sinensis based on ITS sequence and Thitarodes based on COI sequences, respectively, we estimated the time of the most recent common ancestor (TMRCA) for each divergence node, using the commonly used fossil calibration point and nucleotide substitution rate for fungal and insects, respectively. Specifically, for 0 .sinensis, the TMRCA of QLo and TPo was 1.15 Mya (Fig. 1f), coincide with Kunlun-Huanghe movements (1.2-0.6 Mya) (Li and Fang 1998). The TMRCA of all O.sinensis clades was $1.71 \mathrm{Mya}$ (Fig. 1f), parallel to the period of Scene $C$ of Tibetan movements (1.7 Mya) (Li and Fang 1998). Previous study also revealed that population divergence of 0 .sinensis was associated with latitude-based geographical variation (Quan et al. 2014a). These results indicated that the lifestyle of 0 .sinensis might be sensitive to latitude variance and their differentiation was at least partially influenced by Tibetan Plateau uplift exhumation events. The TMRCA estimates for host insects ranged from 0.303 to 0.0007 Mya (Fig. 1g), which were much younger than that of 0 .sinensis. This could be due to the absence of appropriate fossil calibration for insects, hereafter we did not consider the TMRCA of insects.

\section{IBS correlation between fungal and host insect}

Another evidence of coevolution was previously illustrated as a positive correlation between genetic distance and geographic distance within O.sinensis and Thitarodes, respectively (Quan et al. 2014b). However, this could be a consequence of either neutral evolution or other adaptive evolution within each species. A direct evidence for coevolution could be a positive correlation between 0 .sinensis and Thitarodes from different geographic distributions. Here we assessed the identity-by-similarity (IBS) matrix within 0 .sinensis based on ITS sequence in Table S2 and Thitarodes based on COI sequence in Table S3, respectively. Ranked from lowest to highest genetic differentiation between 0 .sinensis within groups are Nyingchi, Yunnan, Qinghai Lake and Tibetan Plateau, while that between host insects within groups are Qinghai Lake, Yunnan, Tibetan Plateau and Nyingchi (Fig. 1h-i), showing that the fungal may not co-evolve with the host insects. 
Parasite and corresponding host driven by arms race should experience similar divergence time and demographic histories. However, the branch length ratio of TPt/NCt (1.04) was nearly 2 times to that of TPo/NCo (0.53). Moreover, BSP inference (Drummond et al. 2005) inferred a conspicuous increase of effective population size in $\mathbf{0}$.sinensis and a moderate reduction in Thitarodes (Fig. $\mathbf{1 j - k}$ ). This discrepancy of dynamic demography further indicated rare influence of arms race on the evolution of interactions between 0 .sinensis and Thitarodes.

\section{Discussion}

Although evidences mentioned above disapproved coevolution between 0 .sinensis based on ITS gene and its host Thitarodes based on COI gene for the major part of their habitats, Qinghai Lake stood out as an exception. QLo formed a unique clade that diverged from the lineage to TPo, after the separation of NCo lineage (Fig. 1d). The same phylogenetic topology occurred in QLt (Fig. 1e). Moreover, the branch length ratio of QLo/NCo was 0.67 in 0 .sinensis, which was comparable to QLt/NCt in host insects (0.71). The IBS of QHo and QHt are higher correlated (Fig. 1I). Additionally, both QLo and QLt showed similarly constant population size since $0.0006 \mathrm{Mya}$ (Fig. 1k). Collectively, these evidences suggested a possibility that there could be some factors affecting both 0 .sinensis and Thitarodes simultaneously and therefore driving similar evolutionary pattern.

As mentioned above, the divergence of 0 .sinensis was sensitive to latitude. Considering that the Qinghai Lake basin was fractured from the northwest to the southeast during the QTP uplift in the late Tertiary (Madsen et al. 2008), it was reasonable to assume that the divergence of QLo was associated with the occurrence of Qinghai Lake. Indeed, we estimated the divergence time of Qinghai Lake group to be 1.15 Mya, which was consistent with the formation of Qinghai Lake (2-0.2 Mya) (Madsen et al. 2008). During the middle Pleistocene, vast crustal movements caused the area to sink substantially, forming the lake (Madsen et al. 2008). The regional climate, rainfall and plant distributions in this area may be shaped by the lake and the topography of the basin. Therefore, both 0 .sinensis and its host insects in this region may evolve parallely due to the long-term geographical isolation under neutral evolution, or even the adaptation to the distinct ecological environment.

In conclusion, we found incongruent phylogenetic topology between 0 .sinensis and host insects Thitarodes from the main distribution of China. This together with varied branch length ratio, different demographic dynamics, and uncorrelated IBS matrix consistently suggested independent evolution between 0 .sinensis and Thitarodes rather than coevolved arms race. The appearance of Qinghai Lake might promoted island isolation and thus drove the divergence of both 0 .sinensis and Thitarodes around Qinghai Lake from other lineages.

In our study, we used the ITS sequence of 0 .sinensis with $504 \mathrm{bp}$ length and COI sequence of Thitarodes with $617 \mathrm{bp}$ length to analyze, leading to the results being considered tentative. However, previous study has revealed that although the sequence is short, the results are relatively reliable if the sample size is 
large enough (Ma et al. 2020). Consequently, our study was limited, but it also provided new insights into host-parasite coevolution.

\section{Declarations}

\section{Funding}

This work was supported by the Strategic Priority Research Program of the Chinese Academy of Sciences (XDA2004010302), Second Tibetan Plateau Scientific Expedition and Research (STEP) Program (2019QZKK0501), National Natural Science Foundation of China (31860305), Sino-Africa Joint Research Center, Chinese Academy of Sciences (SAJC201611), and Research Center for Ecology and Environment of Central Asia, Chinese Academy of Sciences. Y.L. was supported by the Young Academic and Technical Leader Raising Foundation of Yunnan Province.

\section{Conflicts of interest}

The authors declare no competing interest.

\section{Availability of data and material}

All data generated or analyzed during this study are included in this published article and its supplementary information files.

\section{Ethics approval and consent to participate}

Not applicable

\section{Consent for publication}

Not applicable

\section{Contributions}

ST provided the samples used in study; XL conducted the experiments of DNA extraction, amplification and sequencing; ZG analyzed data and wrote the manuscript; $Y L$ provided funds, conceived, and revised the manuscript. All authors read and approved the final manuscript.

\section{Acknowledgements}

This work was supported by the Strategic Priority Research Program of the Chinese Academy of Sciences (XDA2004010302), Second Tibetan Plateau Scientific Expedition and Research (STEP) Program (2019QZKK0501), National Natural Science Foundation of China (31860305), Sino-Africa Joint Research Center, Chinese Academy of Sciences (SAJC201611), and Research Center for Ecology and Environment of Central Asia, Chinese Academy of Sciences. Y.L. was supported by the Young Academic and Technical Leader Raising Foundation of Yunnan Province. Samples used in this study were provided by Prof. T.Sha, 
State Key Laboratory for Conversation and Utilization of Bio-resources in Yunnan, School of Life Sciences, Yunnan University, Kunming, China. We thanked X.X Guo (Henan Univesity, China) for drawing the samples distribution map.

\section{References}

Barido-Sottani J, Bošková V, Plessis LD, Kühnert D, Magnus C, Mitov V, Müller NF, PečErska J, Rasmussen DA, Zhang C, Drummond AJ, Heath TA, Pybus OG, Vaughan TG, Stadler T (2017) Taming the BEAST-A Community Teaching Material Resource for BEAST 2. Systematic Biology 67(1):170-174 https://doi.org/10.1093/sysbio/syx060

Bouckaert R, Heled J, K“"1hnert D, Vaughan T, Wu C-H, Xie D, Suchard MA, Rambaut A, Drummond AJ (2014) BEAST 2: A Software Platform for Bayesian Evolutionary Analysis. Plos Comput Biol https://doi.org/10.1371/journal.pcbi.1003537

Bouckaert R, Vaughan TG, Barido-Sottani J, Duchene S, Fourment M, Gavryushkina A, Heled J, Jones G, Kuhnert D, De Maio N, Matschiner M, Mendes FK, Muller NF, Ogilvie HA, du Plessis L, Popinga A, Rambaut A, Rasmussen D, Siveroni I, Suchard MA, Wu CH, Xie D, Zhang C, Stadler T, Drummond AJ (2019) BEAST 2.5: An advanced software platform for Bayesian evolutionary analysis. Plos Comput Biol 15(4) https://doi.org/10.1371/journal.pcbi.1006650

Brower AV (1994) Rapid Morphological Radiation and Convergence among Races of the Butterfly Heliconius-Erato Inferred from Patterns of Mitochondrial-DNA Evolution. PNAS 91(14):6491-6495 https://doi.org/10.1073/pnas.91.14.6491

Burland TG (2000) DNASTAR's Lasergene sequence analysis software. Methods Mol Biol 132:71-91 https://doi.org/10.1385/1-59259-192-2:71

Drummond AJ, Rambaut A, Shapiro B, Pybus OG (2005) Bayesian coalescent inference of past population dynamics from molecular sequences. Mol Biol Evol 22(5):1185-1192 https://doi.org/10.1093/molbev/msi103

Edgar RC (2004) MUSCLE: multiple sequence alignment with high accuracy and high throughput. Nucleic Acids Res 32(5):1792-7 https://doi.org/10.1093/nar/gkh340

Heled J (2012) Sequence diversity under the multispecies coalescent with Yule process and constant population size. Theor Popul Biol 81(2):97-101 https://doi.org/10.1016/j.tpb.2011.12.007

Heled J, Drummond AJ (2012) Calibrated tree priors for relaxed phylogenetics and divergence time estimation. Syst Biol 61(1):138-49 https://doi.org/10.1093/sysbio/syr087

Joop G, Vilcinskas A (2016) Coevolution of parasitic fungi and insect hosts. Zoology 119(4):350-8 https://doi.org/10.1016/j.zool.2016.06.005 
Kimura M (1980) A Simple Method for Estimating Evolutionary Rates of Base Substitutions through Comparative Studies of Nucleotide-Sequences. J Mol Evol 16(2):111-120 https://doi.org/10.1007/Bf01731581

Kumar S, Stecher G, Li M, Knyaz C, Tamura K (2018) MEGA X: Molecular Evolutionary Genetics Analysis across Computing Platforms. Mol Biol Evol 35(6):1547-1549 https://doi.org/10.1093/molbev/msy096

Kumar S, Stecher G, Suleski M, Hedges SB (2017) TimeTree: A Resource for Timelines, Timetrees, and Divergence Times. Mol Biol Evol 34(7):1812-1819 https://doi.org/10.1093/molbev/msx116

Legendre P, Desdevises Y, Bazin E (2002) A statistical test for host-parasite coevolution. Syst Biol 51(2):217-34 https://doi.org/10.1080/10635150252899734

Li JJ, Fang X (1998) Research on the uplift of the Qinghai-Xizang Plateau and environmental changes. Chinese Sci Bull 43:1569-1574

Li X, Liu Q, Li WJ, Li QP, Qian ZM, Liu XZ, Dong CH (2019) A breakthrough in the artificial cultivation of Chinese cordyceps on a large-scale and its impact on science, the economy, and industry. Crit Rev Biotechnol 39(2):181-191 https://doi.org/10.1080/07388551.2018.1531820

Ma XY, Ning T, Adeola AC, Li J, Esmailizadeh A, Lichoti JK, Agwanda BR, Isakova J, Aldashev AA, Wu SF, Liu HQ, Abdulloevich NT, Afanasevna ME, Ibrohimovich KB, Adedokun RAM, Olaogun SC, Sanke OJ, Mangbon GF, Chen X, Yang WK, Wang Z, Peng MS, Ommeh SC, Li Y, Zhang YP (2020) Potential dual expansion of domesticated donkeys revealed by worldwide analysis on mitochondrial sequences. Zool Res 41(1):51-60 https://doi.org/10.24272/j.issn.2095-8137.2020.007

Madsen DB, Ma HZ, Rhode D, Brantingham PJ, Forman SL (2008) Age constraints on the late Quaternary evolution of Qinghai Lake, Tibetan Plateau. Quaternary Res 69(2):316-325 https://doi.org/10.1016/j.yqres.2007.10.013

Milutinovic B, Peuss R, Ferro K, Kurtz J (2016) Immune priming in arthropods: an update focusing on the red flour beetle. Zoology 119(4):254-261 https://doi.org/10.1016/j.zool.2016.03.006

Neiman M, Fields P (2016) Antagonistic Interspecific Coevolution. Encyclopedia of Evolutionary Biology:93-100 https://doi.org/10.1016/B978-0-12-800049-6.00196-7

Peakall R, Smouse PE (2012) GenAIEx 6.5: genetic analysis in Excel. Population genetic software for teaching and research-an update. Bioinformatics 28(19):2537-2539

https://doi.org/10.1093/bioinformatics/bts460

Pedrini N, Ortiz-Urquiza A, Huarte-Bonnet C, Fan Y, Juárez MP, Keyhani NO (2015) Tenebrionid secretions and a fungal benzoquinone oxidoreductase form competing components of an arms race between a host and pathogen. PNAS 112(28):E3651-E3660 https://doi.org/10.1073/pnas.1504552112 
Peng QY, Zhong X, Lei W, Zhang GR, Liu X (2013) Detection of Ophiocordyceps sinensis in soil by quantitative real-time PCR. Can J Microbiol 59(3):204-209 https://doi.org/10.1139/cjm-2012-0490

Quan QM, Chen LL, Wang X, Li S, Yang XL, Zhu YG, Wang M, Cheng Z (2014a) Genetic Diversity and Distribution Patterns of Host Insects of Caterpillar Fungus Ophiocordyceps sinensis in the Qinghai-Tibet Plateau. Plos One 9(3) https://doi.org/10.1371/journal.pone.0092293

Quan QM, Wang QX, Zhou XL, Li S, Yang XL, Zhu YG, Cheng Z (2014b) Comparative Phylogenetic Relationships and Genetic Structure of the Caterpillar Fungus Ophiocordyceps sinensis and Its Host Insects Inferred from Multiple Gene Sequences. J Microbiol 52(2):99-105 https://doi.org/10.1007/s12275-014-3391-y

Rambaut A, Drummond AJ, Xie D, Baele G, Suchard MA (2018) Posterior Summarization in Bayesian Phylogenetics Using Tracer 1.7. Syst Biol 67(5):901-904 https://doi.org/10.1093/sysbio/syy032

Rozas J, Ferrer-Mata A, Sanchez-DelBarrio JC, Guirao-Rico S, Librado P, Ramos-Onsins SE, SanchezGracia A (2017) DnaSP 6: DNA Sequence Polymorphism Analysis of Large Data Sets. Mol Biol Evol 34(12):3299-3302 https://doi.org/10.1093/molbev/msx248

Sung GH, Hywel-Jones NL, Sung JM, Luangsa-Ard JJ, Shrestha B, Spatafora JW (2007) Phylogenetic classification of Cordyceps and the clavicipitaceous fungi. Stud Mycol(57):5-59 https://doi.org/10.3114/sim.2007.57.01

Sung GH, Poinar GO, Jr., Spatafora JW (2008) The oldest fossil evidence of animal parasitism by fungi supports a Cretaceous diversification of fungal-arthropod symbioses. Mol Phylogenet Evol 49(2):495-502 https://doi.org/10.1016/j.ympev.2008.08.028

Wang JB, Elya C, St Leger RJ (2020) Genetic variation for resistance to the specific fly pathogen Entomophthora muscae. Sci Rep 10(1):14284 https://doi.org/10.1038/s41598-020-71262-w

Wang XL, Yao YJ (2011) Host insect species of Ophiocordyceps sinensis: a review. Zookeys(127):43-59 https://doi.org/10.3897/zookeys.127.802

White T, Bruns T, Lee S, Taylor J, Innis M, Gelfand D, Sninsky J (1990) Amplification and Direct Sequencing of Fungal Ribosomal RNA Genes for Phylogenetics PCR Protocols. vol 31, pp 315-322

Zhang Y, Li E, Wang C, Li Y, Liu X (2012) Ophiocordyceps sinensis, the flagship fungus of China: terminology, life strategy and ecology. Mycology 3(1):2-10 https://doi.org/10.1080/21501203.2011.654354

Zhang Y, Zhang S, Li Y, Ma S, Wang C, Xiang M, Liu X, An Z, Xu J, Liu X (2015) Phylogeography and evolution of a fungal-insect association on the Tibetan Plateau. Mol Ecol 23(21):5337-5355 https://doi.org/10.1111/mec. 12940 


\section{Figures}
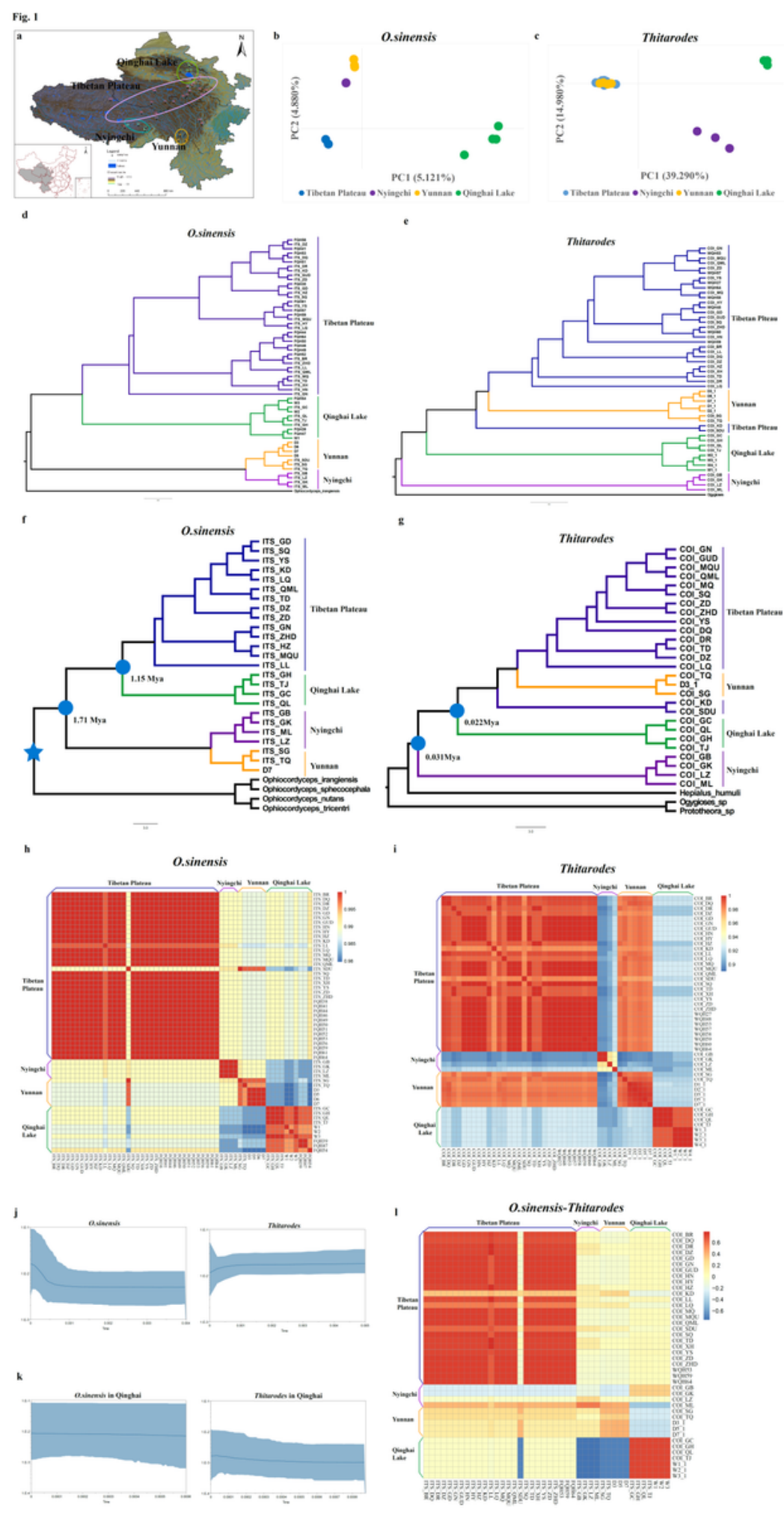

Figure 1

The phylogenetic and evolutionary relationship between 0.sinensis and host insects. a Distribution of Ophiocordyceps sinensis samples used in the study. The pink ellipse represents the interior and northeast of Tibetan Plateau; the light blue ellipse represents Nyingchi; The green ellipse represents the north of 
Qinghai (around the Qinghai Lake); and the orange ellipse represents Yunnan. b, c Principal components analysis (PCA) showing PC1 against PC2 using SNP of ITS and COI to show the genetic structure of O.sinensis (left) and host insects (right), respectively. $d$, e The phylogenetic tree of O.sinensis constructed by ITS dataset (left) and the phylogenetic tree of host insects constructed by COI dataset (right) using Maximum Likelihood method. $f$ Estimation of the divergence time for O.sinensis based on the ITS data set. The fossil evidence of Paleoophiccordyceps coccophagus reported by Sung et al. (2008) was used as a crown calibration point for Ophiocordyceps (star). The nodes indicate divergence time between populations. g Estimation of divergence time for host insects based on the COI dataset. The age of host insects was calibrated by the standard insect mtDNA clock. The nodes indicate divergence time between populations. $\mathrm{h}$ Heatmap of the relationship within and among O.sinensis from different regions based on IBS distance as shown by the color scale bar on the right. i Heatmap of the relationship within and among host insects from different regions based on IBS distance as shown by the color scale bar on the right. Each cell represents an individual of the O.sinensis and host insects. j, k Demographic inference inferred by coalescent-based Bayesian skyline plots. j Bayesian skyline plots (BSP) of O.sinensis (left) and host insects (right). $k$ Bayesian skyline plots (BSP) of O.sinensis (left) and host insects (right) in Qinghai Lake. The X-axis shows time in millions of years before present. The Y-axis (logarithmic scale) indicates effective population size estimates multiplied by generation time. The dashed line represents the median estimate of the effective population size, with the $95 \%$ high posterior density interval shown in blue. I IBS correlation was assessed using Spearman method between O.sinensis and corresponding host insects (seen in Table S4 and S5). The heatmaps of IBS correlation between each fungal sample and corresponding infected insect based on IBS distance as shown by the color scale bar on the right. Each cell represents an individual of the O.sinensis ( $x$ axis) and corresponding host insects ( $y$ axis). *The abbreviation of samples was given in Table S1.

\section{Supplementary Files}

This is a list of supplementary files associated with this preprint. Click to download.

- SupplementaryTable.xlsx 